\title{
Caractéristiques de reproduction d'un troupeau de vaches créoles élevées en zone tropicale
}

\author{
D. GAUTHIER, A. XANDE \\ avec la collaboration technique de A. Grude \\ I.N.R.A., Station de Recherches zootechniques \\ Centre de Recherches Antilles-Guyane, Domaine Duclos, 97170 Petit-Bourg, Guadeloupe
}

\begin{abstract}
Résumé
A partir de l'enregistrement systématique des dates de mise-bas du troupeau de vaches créoles de la Station de Recherches zootechniques de l'I.N.R.A. en Guadeloupe, nous avons analysé la répartition mensuelle des fécondations, de la fertilité et de l'intervalle entre vélages, paramètres caractérisant la fécondité.

Les femelles, entretenues sur savane naturelle à dominante de Dicanthium caricosum, sont laissées, à partir de $250 \mathrm{~kg}$, en permanence avec un taureau.

Bien que les mise-bas aient lieu toute l'année, les fécondations sont plus fréquentes entre août et novembre (44 p. 100). Mensuellement, le pourcentage de fécondations et la fertilité sont positivement corrélés avec la pluviométrie et sont les plus élevés pendant la période de reprise de poids, en début de saison humide.

La durée moyenne de l'intervalle entre deux vélages consécutifs est de $496 \pm 182$ jours, ce qui traduit un intervalle moyen vélage-fécondation d'environ 7 mois. La moyenne des intervalles de durée inférieure à 700 jours est de $437 \pm 106$ jours. La durée de ce type d'intervalle varie avec le rang de vélage et avec la saison, en liaison avec la pluviométrie du mois suivant la mise-bas.
\end{abstract}

L'âge au premier vélage est de 36 mois, correspondant à une fécondation à 27 mois.

\section{Introduction}

En Guadeloupe, les bovins frisons ont une meilleure fertilité pendant la saison fraîche, de décembre à avril, que pendant le reste de l'année (VIVIER, MichaleTDoreau \& Grude, 1975). La température semble être le facteur du milieu responsable de cette variation. Quant aux bovins créoles, animaux élevés pour la production de viande, leurs caractéristiques de reproduction et les facteurs qui en limitent l'efficacité sont peu connus. C'est pourquoi nous avons analysé, à partir des enregistrements effectués sur un troupeau de vaches créoles quelques paramètres intervenant dans la productivité d'un élevage : âge au premier vélage, intervalle entre vélages, intervalle vélage-fécondation et répartition des mise-bas. De plus nous avons étudié les relations entre ces critères et les caractéristiques du milieu d'élevage. 


\section{Matériel et méthodes}

Le troupeau de vaches créoles de la Station de Recherches zootechniques de I'I.N.R.A. en Guadeloupe (Domaine de Gardel) est le support de cette étude. Il a été constitué dès 1969 à partir des animaux achetés dans les élevages de l'île. L'effectif varie entre 30 et 60 femelles reproductrices de 1971 à 1978.

\section{Situation géographique}

L'élevage est situé dans la zone sèche et calcaire de la Guadeloupe $\left(\begin{array}{ll}16^{\circ} & 10 \text { de }\end{array}\right.$ latitude nord et $61^{\circ} 40$ de longitude ouest). La moyenne annuelle des précipitations enregistrées quotidiennement sur le domaine, pendant la durée de l'étude est de $1280 \mathrm{~mm}$. En dépit des variations annuelles importantes, une saison des pluies (août à décembre : $810 \mathrm{~mm}$, soit $162 \mathrm{~mm} / \mathrm{mois}$ ), et une saison sèche (janvier à juillet : $470 \mathrm{~mm}$ soit $67 \mathrm{~mm} /$ mois) peuvent être définies. Les températures maximales moyennes varient entre $27{ }^{\circ} \mathrm{C}$ (janvier) et $32{ }^{\circ} \mathrm{C}$ (août) et les minimales entre $21^{\circ} \mathrm{C}$ et $25^{\circ} \mathrm{C}$. L'hygrométrie moyenne est toujours supérieure à 70 p. 100 . La durée de la période claire du nycthémère passe au cours de l'année, de 11 heures (fin décembre) à 13 heures (fin juin).

\section{Animaux}

Les animaux exploitent une savane naturelle à dominante de Dicanthium caricosum dans des conditions proches de celles pratiquées en Guadeloupe et en Martinique. Ils disposent d'eau en permanence et sont détiqués régulièrement. Le chargement moyen des pâturages est de 2 à 3,5 vaches/ha. Aucun aliment extérieur n'est apporté, sauf en cas d'extrême sécheresse.

Les veaux sont sevrés au poids approximatif de $120 \mathrm{~kg}$. Les génisses sont mises à la reproduction à un poids d'environ $250 \mathrm{~kg}$. La monte, naturelle et libre, est assurée par des taureaux créoles laissés en permanence dans les troupeaux à raison d'un mâle pour 30 à 40 femelles. Aucune politique de réforme systématique n'est définie.

De 1971 à 1978, 119 vaches, dont 61 d’âge inconnu, ont effectué 320 mise-bas. Vingt et une femelles présentes pendant trois années consécutives $(1974,1975,1976)$ ont été pesées tous les mois.

\section{Méthodes d'analyse}

Le pourcentage mensuel de mise-bas est le nombre de mise-bas observées pendant le mois sur le nombre total dans l'année multiplié par 100. En supposant une durée de gestation fixe de 283 jours (Gauthier et al., données non publiées), il est possible de déduire la répartition des fécondations de celle des mise-bas. Mais cette donnée, qui dépend du nombre mensuel de femelles susceptibles d'être fécondées, ne donne qu'une appréciation partielle de la variation de la fertilité. C'est pourquoi nous avons défini la fertilité mensuelle, exprimée en p. 100. Elle correspond au nombre de femelles multipares fécondées pendant le mois considéré (chiffre déduit 
des dates de mise-bas), divisé par le nombre de femelles multipares susceptibles d'être fécondées pendant cette période. Une femelle est considérée comme susceptible d'être fécondée à partir du mois civil qui suit celui de son vélage.

Les relations entre les variables sont décrites par le coefficient de corrélation de rang de Spearman. La variation de la durée de l'intervalle entre vélages est étudiée par l'analyse de la variance après une transformation logarithmique de cette variable de façon à normaliser sa loi de distribution. L'uniformité de la répartition des pourcentages mensuels de fécondation ou de fertilité est vérifiée par le test de $\mathrm{X}^{2}$ (SNEDECOR \& Cochran, 1971).

\section{Résultats}

\section{Distribution annuelle des vélages et des fécondations}

Les vélages ont lieu toute l'année; cependant la distribution est significativement différente d'une distribution uniforme $(p<0.01)$. En effet, en moyenne 44 p. 100 des mise-bas ont lieu du mois de mai au mois d'août, correspondant à un pic de fécondations entre les mois d'août et novembre (fig. 1).

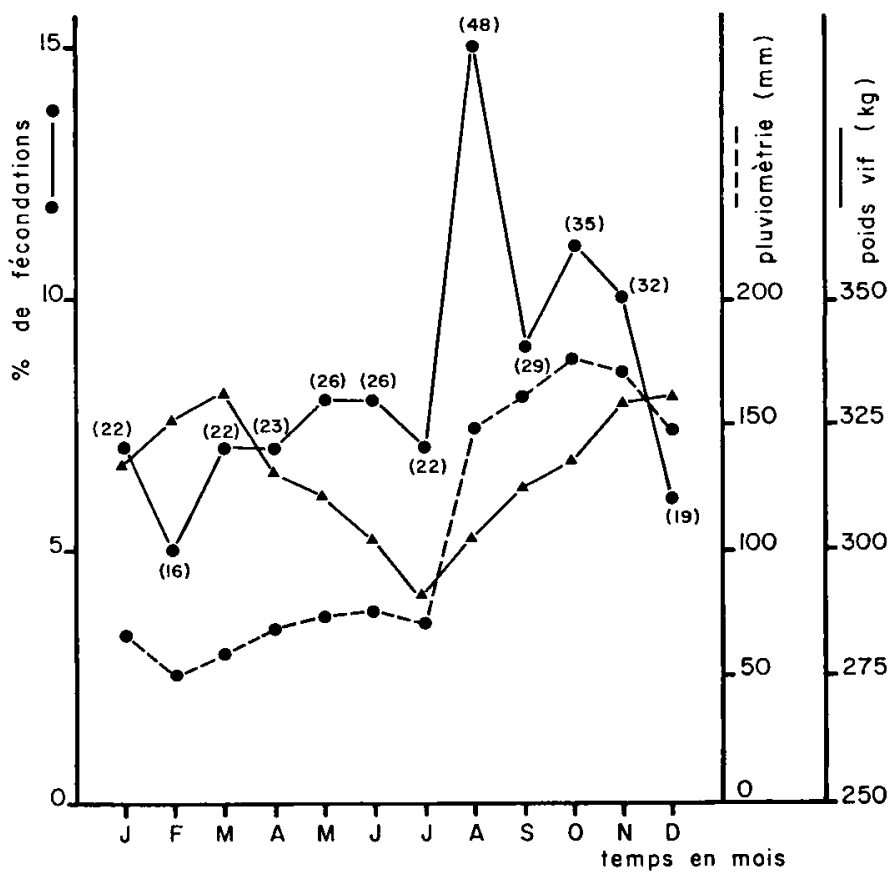

Fig. 1

Variations mensuelles moyennes du poids vif et du pourcentage de fécondation d'un troupeau de vaches créoles et de la pluviométrie de la zone d'élevage. Monthly mean variations of live weight and fertilization rate of a Creole cow herd as well as of the pluviometry in the production area.

( ) = Nombre de femelles fécondées.

Number of fertilized females. 
Les variations mensuelles de la pluviométrie et du pourcentage de fécondations sont importantes d'une année à l'autre; mais ces deux variables sont corrélées linéairement $(r=0,76, p<0,001)$ pendant toute la durée de l'étude. En moyenne, sur les 7 ans, une corrélation de rang de $0,74(\mathrm{p}<0,01)$ existe entre la pluviométrie mensuelle et le pourcentage de fécondations du mois considéré (fig. 1). Les autres paramètres climatiques : température, humidité, photopériode, n'influencent pas significativement ce pourcentage. Pendant la saison humide (août à décembre) et au début de la saison sèche (janvier à mars) le poids vif apparent des femelles croît régulièrement. Après cette date, les vaches perdent du poids jusqu'à la reprise des pluies (août), période correspondant au maximum de fécondations.

\section{Variation annuelle de la fertilité}

La fertilité mensuelle moyenne est toujours différente de zéro et varie significativement au cours de l'année $(\mathrm{p}<0,01)$. Ainsi elle est plus élevée pendant la saison des pluies (août à décembre; 25,4 p. 100) que pendant la saison sèche (janvier à juillet ; 14,7 p. $100 ; \mathrm{p}<0,05$; fig. 2).

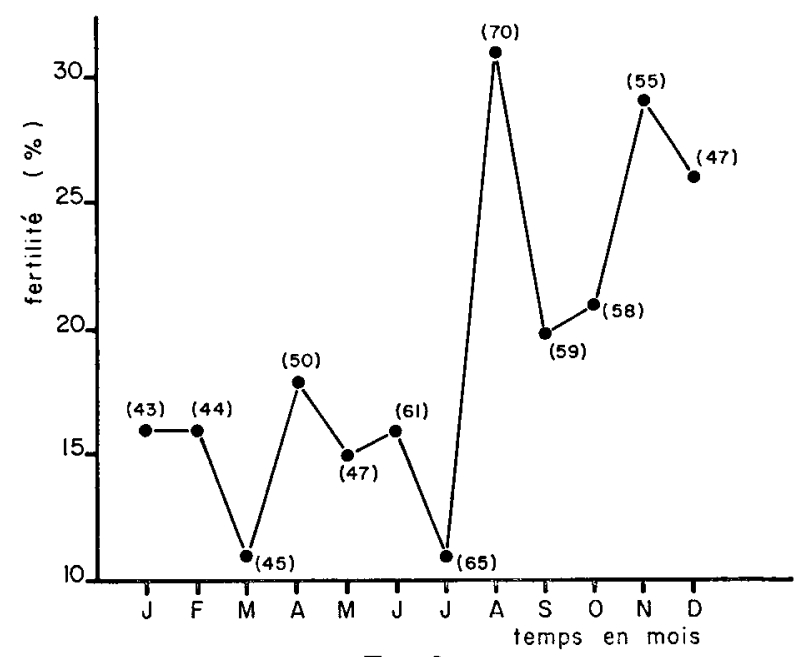

FIG. 2

Variations mensuelles de la fertilité d'un troupeau de vaches créoles.

Monthly variations in the fertility of a Creole cow herd.

( ) = Nombre de femelles susceptibles d'être fécondées.

Number of females liable to be fertilized.

\section{Intervalle entre vélages}

L'intervalle moyen calculé à partir de 173 données est de $496 \pm 182$ jours $(\mathrm{m} \pm \sigma)$; l'intervalle vélage-fécondation est donc en moyenne de 7 mois.

Vingt-neuf p. 100 des intervalles entre vélages ont une durée inférieure ou égale à 365 jours; 13,8 p. 100 ont une durée supérieure ou égale à 700 jours. 
La variable a une répartition bimodale ; la moyenne de la première classe, c'est-à-dire des intervalles de durée inférieure à 700 jours, est de $437 \pm 106$ jours $(m \pm \sigma)$. Par la suite, seule cette classe est considérée.

La durée de l'intervalle entre vélages varie en fonction du mois de mise-bas de 389 à 507 jours (fig. 3). Elle est reliée à la pluviométrie du mois suivant la mise-bas (coefficient de corrélation de rang, $\mathrm{r}=0,80 ; \mathrm{p}<0,01$ ). En revanche, les pluviométries du mois de la mise-bas et du mois précédent ne sont pas reliées à la durée de cet intervalle.

L'intervalle entre vélages diminue significativement avec l'augmentation de la parité. Il est de 19,5 mois entre la $1^{\text {re }}$ et la $2^{\mathrm{e}}$ mise-bas, 15,8 mois entre la $2^{\mathrm{e}}$ et la $3^{\mathrm{e}}$, 14,1 mois entre la $3^{e}$ et la $4^{e}$ et 13 mois au-delà.

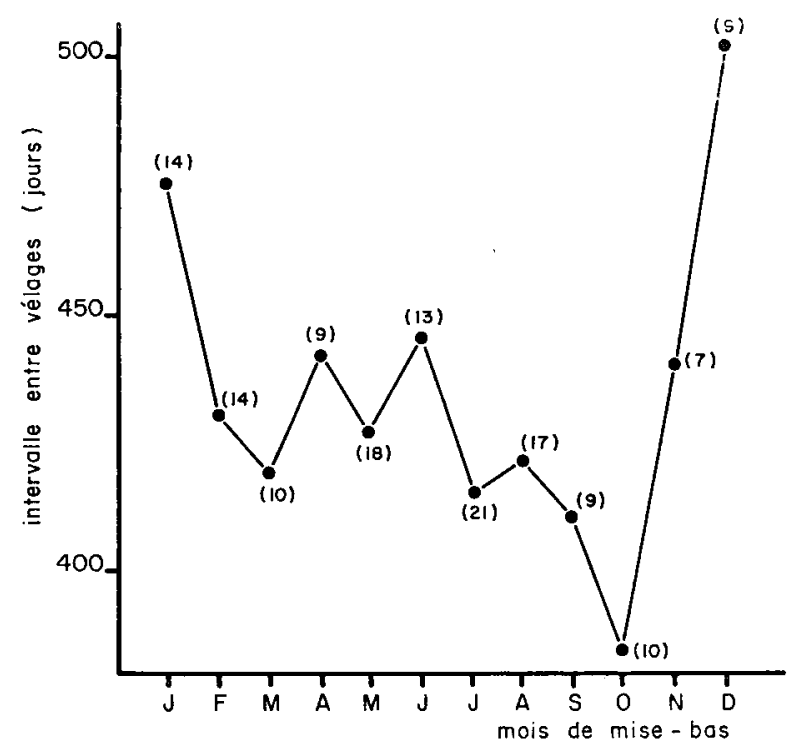

FIG. 3

Variations mensuelles de la durée de l'intervalle entre les vélages de rang $i$ et $i+1$ en fonction du mois de vélage de rang $i$.

Monthly variations in the length of the interval between calvings of the order $i$ and $i+1$ according to calving month of order $i$.

( ) = Nombre d'observations.

Number of observations.

\section{Premier vélage}

L'âge au premier vélage est de $36 \pm 4,3$ mois et correspond à une première fécondation réalisée à 26,6 mois. uniforme.

La répartition annuelle des premiers vélages n'est pas différente d'une répartition 


\section{Discussion}

L'indice de fertilité mensuelle tel qu'il est défini dans notre travail permet de mieux préciser la variation de fertilité que l'étude de la répartition des mise-bas. En fait il supprime le biais dû à la variation du nombre de femelles susceptibles d'être fécondées. Ainsi le faible pourcentage de fécondations pendant le mois de décembre n'est pas lié à une subfertilité de la vache créole à cette période mais au nombre réduit de femelles susceptibles d'être fécondées (fig. 2). Si les études antérieures réalisées chez les bovins (OrTavant, Mauleon \& Thibault, 1964 ; Landais, Poivey \& Seitz, 1980; Mauleon, 1980) mettent en évidence une période pendant laquelle les fécondations sont peu nombreuses, elle ne permettent pas de faire ce type d'analyse.

Chez les bovins créoles, il existe une variation saisonnière de la fertilité. Elle est en liaison avec la pluviométrie, comme dans le cas du Zébu Gobra au Sénégal (Denis \& Thiongane, 1973). Or la quantité de pluie est le principal facteur régulant la pousse de l'herbe (Doreau \& Vivier, 1977; Gravelier, résultats non publiés) et par conséquent les disponibilités alimentaires et la variation du poids vif des vaches. De mars à juillet, la perte de poids des animaux s'accompagne d'une fertilité faible ; en revanche, dès que le poids des femelles augmente (août) la fertilité s'accroît. Cette relation entre disponibilités alimentaires et variation des fécondations est connue dans des troupeaux de bufflesses maintenues en permanence avec le taureau (BuvANENDRAN, JALATGE \& GANESAN, 1971) et une telle concordance entre dynamique du poids et fertilité a déjà été observée au cours d'une saison de monte (YoudAN \& King, 1977 ; Grosskopf, 1978).

Néanmoins, notre étude ne permet pas de dissocier les parts respectives du mâle et de la femelle dans cette variation.

La variation saisonnière de l'intervalle mise-bas - fécondation, également en relation avec la pluviométrie, s'explique de la même façon que celle de la fertilité, comme cela a été décrit par Rao \& Reddy (1967) et Dhillon et al. (1970). Le niveau d'alimentation pendant le début de la lactation semble être le facteur primordial, l'alimentation pendant la fin de gestation n'ayant que peu d'influence, puisque seule la pluviométrie du mois suivant la mise-bas est reliée à la durée de cet intervalle.

L'intervalle moyen entre vélages (496 jours) est long. En effet les valeurs relevées dans les différents travaux effectués en zone tropicale sont inférieures, soit 409, 416, 437, 473 jours respectivement pour les races Brahman (Plasse, Koger \& Warnick, 1968), Sahiwal, Red Sindhi (Johar \& TaYlor, 1967) et Gobra (DENIS, 1971).

Mais dans la plupart des troupeaux utilisés pour les études précédentes, une politique de réforme ayant pour effet d'éliminer les intervalles de durée importante, était appliquée. En rejetant les intervalles entre vélages, supérieurs à 700 jours (considérés comme «anormaux»), la moyenne obtenue (437 jours) est alors comparable aux données ci-dessus. Toutefois cette valeur reste élevée, en comparaison des résultats obtenus en zone tempérée et avec des races taurines (AURIOL, 1955; VisSAC \& Poly, 1957). La présence du mâle en permanence dans le troupeau (Donaldson, 1962) et les mauvaises conditions alimentaires (GrosskopF, 1978) peuvent rendre compte d'une partie de ces différences. 
La diminution de l'intervalle entre vélages avec augmentation de la parité est aussi un résultat fréquemment démontré : Lewis \& Horwood (1950) ; Plasse, Koger \& Warnick (1968); Denis (1971). Elle peut être attribuée à un anœstrus post partum généralement plus court chez la multipare que chez la primipare (TERQu et al., 1981).

La fonction de reproduction de la vache créole se révèle donc peu sensible à une température élevée. En effet sa fertilité est bonne pendant la saison chaude et humide.

Accepté pour publication en avril 1982.

\section{Summary}

\section{Reproductive performance of Creole cow herd in a tropical area}

On the basis of systematic recordings of calving dates in Creole cows kept at the Experimental Research Station of I.N.R.A., Guadeloupe, we analysed the annual distribution of conceptions, fertility and calving interval, characterizing the fertility of these cows.

Calvings took place all year round, but the percentage of conceptions was higher between August and November (44 p. $100 ;$ p $<0.05$, fig. 1). Monthly fertility defined as the ratio of the number of fertilized females per month to the number of females liable to be fertilized, multiplied by 100 showed a peak from august to december. The monthly conception rate and fertility were high during the rain season and when the cows gained in weight (fig. 1).

The interval between two successive calvings was $496 \pm 182$ days corresponding to a mean calving-conception interval of 7 months. The mean length of intervals shorter than 700 days was $437 \pm 106$ days. The length of that type of interval decreased with calving order and varied with the season (fig. 3) in positive correlation with the pluviometry during the month after calving.

Age at first calving was 36 months, the first conception was thus obtained at 26.6 months.

\section{Références bibliographiques}

Auriol P., 1955. Influence du mois de vêlage sur la production laitière des vaches Pie rouge de l'Est, dans le Jura. Ann. Zootech., 1, 189-200.

Buvanendran V., Jalatge E.F.A., Ganesan K.N., 1971. Influence of season on the breeding pattern of buffaloes in Ceylan. Trop. Agric. (Trinidad), 48, 97-102.

DENis J.P., 1971. L'intervalle entre les vêlages chez le zébu Gobra (Peulh sénégalais). Rev. Elev. Méd. vét. Pays trop., 24, 635-647.

Denis J.P., Thiongane A.I., 1973. Caractéristiques de la reproduction chez le zébu étudiées au C.R.Z. de Dahra. Communication aux VIII ${ }^{\text {ss }}$ journées médicales de Dakar (9-14 avril).

Dhillon J.S., Acharya R.M., Tiwana M.S., Aggarwal S.C., 1970. Factors affecting the interval between calving and conception in hariana cattle. Anim. Prod., 12, 81-87.

Donaldson L.E., 1962. Some observations on the fertility of beef cattle in North Queensland. Aust. Vet. J., 38, 447-454.

Doreau M., Vivier M., 1977. La prairie naturelle à Dicanthium caricosum en Guadeloupe. I - Effet de la saison sur la production, la composition chimique et la digestibilité in vitro. Agron. trop., 34, 356-361. 
Grosskopf J.F.W., 1978. Non pathogenic factors associated with the reconception of beef cows under extensive conditions. Wld. Congr. Anim. Prod., Buenos Aires (in press).

Johar K.S., TAYLoR C.M., 1967. Calving intervals in Sahiwal and Red Sindhi cows, J.N.K.V.V. Res. J., 1, 44-47.

Landais E., Poivey J.P., Seitz J.L., 1980. Recherches sur la reproduction du cheptel taurin sédentaire du Nord de la Côte-d'Ivoire : utilisation des intervalles entre vêlages; aspects méthodologiques et premiers résultats. Rev. Elev. Med. vét. Pays trop., 33, 193-204.

LEwis R.C., Horwood R.E., 1950. The incidence of age, level of production and management in the calving interval. Q. Bull. Mich. Agric. Exp. Stn., 32, 546-549.

Mauleon P., 1980. Variations circannuelles de la fertilité chez les mammifères domestiques, in : «Rythmes et Reproduction», 15-32, Masson, Paris.

Ortavant R., Mauleon P., Thibault C., 1964. Photoperiodic control of gonadal and hypophyseal activity in domestic mammals. Ann. N.Y. Acad. Sci., 117, 157-192.

Plasse D., Koger M., Warnick A.C., 1968. Reproductive behavior of Bos indicus females in a subtropical environment. III - Calving intervals, intervals from first exposure to conception and intervals from parturition to conception. J. Anim. Sci., 27, 105-112.

Rao A.R., RedDY K.K., 1967. Breeding season in Ongole cows. Indian Vet. J., 46, 679-684.

SADA I., 1968. The length of the gestation period, calving interval and service period in indigenous West African cattle : N'Dama, West African Shorthorn and Sokoto Gudale. Ghana J. Agric. Sci., 1, 91-97.

SNedecor G.W., Cochran W.G., 1971. Méthodes statistiques, traduction de «Statistical methods $\gg, 6^{\circ}$ édition, éd. A.C.T.A., Paris.

Terqui M., Chupin D., Gauthier D., Perez N., Pelot J., Mauleon P., 1981. Influence of management and nutrition on post partum endocrine function and ovarian activity in cows. C.E.E. Congress, Munich (in press).

VissaC B., Poly J., 1957. Etude statistique des causes de variation de quelques paramètres du cycle de reproduction des vaches laitières. II - L'intervalle vêlage - fécondation dans les troupeaux du département de Seine-et-Marne. Ann. Zootech., 2, 237-268.

Vivier M., Michalet-Doreau B., Grude A., 1975. La conduite d'un troupeau laitier intensif en zone tropicale humide (Antilles françaises). Nouv. agron. Antilles-Guyane, 1, 307-321.

Youdan P.G., KING O., 1977. The effect of body weight changes on fertility during the post partum period in dairy cows. Br. Vet. J., 133, 635, 641. 